\title{
Distribution management system framework based on security region for future low carbon distribution systems
}

\author{
Jun XIAO' ${ }^{1}$, Qibo HE ${ }^{1}$, Guoqiang ZU ${ }^{1}$
}

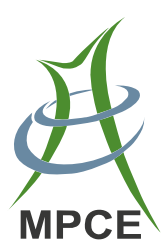

\begin{abstract}
In this paper, a new Distribution Management System (DMS) framework based on security region is proposed. First, the concept of Distribution System Security Region (DSSR) is introduced. DSSR is capable to describe the $N-1$ security boundary of the whole distribution network, including the secure output range of DGs. This new theoretic tool provides a chance for the implementation of real-time security analysis and active controls in DMS. Second, this paper proposes and describes five security states for distribution system. Third, an upgraded DMS enhanced with DSSR is proposed, which consists of advanced security functions such as preventive and predictive control of the trajectory of operating points. Finally, a practical case is presented to simulate the proposed DSSR-enhanced DMS, in which both the security region of network and the output range of DGs are calculated. Typical security functions are also demonstrated. In conclusion, the new DMS framework aims to help operate the system closed to its security boundary in order to improve the efficiency significantly within same security standard. This work is beneficial for future low carbon distribution systems with high penetration rate of DGs.
\end{abstract}

CrossCheck date: 17 December 2014

Received: 20 October 2014 / Accepted: 21 December 2014 / Published online: 27 October 2015

(C) The Author(s) 2015. This article is published with open access at Springerlink.com

$凶$ Jun XIAO

xiaojun@tju.edu.cn

Qibo HE

heqibo@tju.edu.cn

Guoqiang ZU

zuguoqiang_tju@163.com

1 Key Laboratory of Smart Grid of Ministry of Education, Tianjin University, Tianjin, China
Keywords DMS, Security region, $N-1$ security, Distributed generation, Preventive control, Low carbon

\section{Introduction}

Climate change is recognized as one of the key challenges the world is facing in the 21 st century. The measures of reduction of greenhouse gas emissions affect all economic sectors, but, due to its relevant emission levels, the electricity sector plays an important role in this strategy [1-3].

In the whole electricity power system, distribution system plays an important role in reduction of greenhouse gas emissions. On one hand, flexible network configuration and operation mode under distribution automation (DA) provide the great potential to improve operation efficiency and assets utilization resulting in less grid construction [4, 5]; on the other hand, smart grid introduces large amounts of renewable distributed generations (DG) into the distribution systems. These DGs produce far less $\mathrm{CO}_{2}$ than traditional thermal power, and may reduce power losses [6-8].

However, DA and DGs also make the distribution network more complicated in operation. Efficiency and security is difficult to be balanced without proper management approach [4-7]. Although the problems aroused by DGs and electric vehicles have been studied in the research of Active Distribution Network (ADN) [8-11], on-line security assessment and control is rarely involved.

Distribution Management System (DMS) is an effective tool for operation. The traditional DMS has some defects in security control [12-14]:

1) Lack of preventive control approach. Traditional $N-1$ simulation cannot continuously offer the relative position of current operating points to security 
boundary. Thus, dispatchers have not enough predictable information to make decisions [15].

2) Security analysis used only off-line. Traditional $N-1$ simulation needs repeatedly to perform power flow calculation on each component, which is time-consuming for on-line demand.

3) Low efficiency in asset utilization. With lower level of DA, load of faulted substation transformer can be only transferred in the same substation, which means that inter-connected medium-voltage network has not been fully utilized.

4) DG management. Output range of DGs is usually determined by voltages and frequency constraints. This decision method has not been analyzed under $N-1$ security guidelines from the whole system aspect.

A new proposed concept of Distribution System Security Region (DSSR) [16-18] may give new ideas for DMS to overcome the defects above. First, security boundary of a distribution system can be calculated off-line through DSSR theory, which describes the $N-1$ limit of a distribution system. Second, this boundary can be used as the criterion for fast security assessment, which provides a condition for on-line real-time security analysis. For instance, the information on security margins of each feeder can be obtained, thus, dispatchers can obtain the global information of the system by DSSR visualization technology to make effective preventive control decisions. Third, the security margin gives reference of DGs output range with consider of their contribution to system security. This paper applies DSSR to existing DMS to form a new framework focused on efficiency and security, which helps to operate the system closed to its security boundary.

At present, large-scale construction of distribution automation is undergoing in many urban areas in China, which will provide infrastructures for application of DSSR theory to DMS $[4,5]$. A new DMS framework based on DSSR for future low carbon distribution networks is proposed in this paper. A practical case demonstrates the advanced security functions of future DMS.

\section{DSSR theory for distribution network}

The concept of DSSR originates from security region of transmission system [19]. The 'region' method can give systematic and global information about the feasible operation region, which has convinced advantages over the 'point-wise' method. It can provide operators with the relative location of a point in the region and other necessary operating information. Moreover, it can be calculated off-line and applied on-line to determine whether an operating point is secure [16], which can reduce computational burden of security assessment. The DSSR is defined as the set of all operating points that make the distribution system $N-1$ secure, which takes into account the capacity of substation transformers and feeders, network topology and operational constraints $[16,20]$. According to [17], the DSSR model can be mathematically formulated as

$\Omega_{D S S R}=\{\boldsymbol{W} \mid h(x) \leq 0 \quad g(x)=0\}$

where $\boldsymbol{W}=\left(L_{F_{1}}, L_{F_{2}} \cdots L_{F_{n}}\right)^{\mathrm{T}}$ is the operating point, and $L_{F_{i}}$ represents the load of feeder $i$. The inequality and equality constrains is such that

$t_{m n}+L_{F_{n}} \leq r_{n}(\forall m, n)$

$T_{i j}=\sum_{m \in \Omega_{1}^{(i)}, n \in \Omega_{1}^{(j)}} t_{m n}$

$P_{i}=\sum_{m \in \Omega_{1}^{(i)}} L_{F_{m}}(\forall i)$

$T_{i j}+P_{j} \leq R_{j}(\forall i, j)$

where $t_{m n}$ is the load that is transferred from feeder $m$ to feeder $n$ when an $N-1$ fault occurs at outlet of feeder $m$; $r_{n}$ is the rated capacity of feeder $n ; T_{i j}$ is the load that is transferred from transformer $i$ to transformer $j$ when an $N-1$ fault occurs at transformer $i ; \Omega_{1}^{(i)}$ is the set of feeders that derive from transformer $i ; P_{i}$ is all the load supplied by transformer $i ; m \in \Omega_{1}^{(i)}$ means that feeder $m$ derives from the corresponding bus of transformer $i ; R_{i}$ is the rated capacity of transformer $i$.

DSSR and its boundary have three characters [17]: 1) the security boundary is linear and composed of some hyperplanes, which can be fast calculated. 2) DSSR is dense inside. Thus, if the operating point is inside the DSSR, it is secure. Otherwise, it is insecure. Moreover, the location of an operating point in DSSR can be expressed as the distance from the operating point to all the security boundaries, abbreviated as SD (security distance). If SD is positive, the operating point is secure. Otherwise, it's insecure. The greater the positive SD is, the securer the operating point is. Also the greater the negative distance is, the more insecure the operating point is. 3) The dimension of the DSSR can be reduced to 2 or 3 by DSSR visualization technology, which makes the location of the operating points visualized. So operators can supervise the security state of the grid and the security margin of each direction conveniently [18].

Based on the model and characters above, real-time security monitoring can be performed. The location of the operating point in the DSSR can be adjusted to make it $N-1$ secure by adjusting the load distribution among 
substation transformers and feeders. The DSSR theory provides new tool for real-time security monitoring, assessment and control.

\section{Division of security states}

The security control of distribution system should establish a system similar to Dy Liacco security framework for transmission system, which can perform real-time monitoring, alarming, control and optimization. Thus, the distribution system can be decomposed into following operating states, as is shown in Fig. 1.

1) Secure state. The distribution system matches all operating constraints and passes all $N-1$ tests, which means that any single fault in primary feeders or substation transformers will lead to a service interruption in the faulted section only, and all the other affected loads will be restored immediately without violating any operating constraints. As for DSSR, the operating point is inside the boundary of DSSR.

2) Insecure state. The distribution system matches all operating constraints but cannot pass all the $N-1$ tests. As for DSSR, the operating point is outside the boundary of DSSR.

3) Secure and efficient state. Based on the secure state, the load distribution uniforms with the network structure and equipment capacity further and the security margin of each feeders and substation transformers is maximum and well-balanced.

The three states above are normal, on which conditions the distribution system matches all operating constraints. While the following states are abnormal, on which conditions the distribution system doesn't match all operating constraints.

4) Emergency state. In this state, there exists fault, critical overload or overload duration exceeding the limit time.

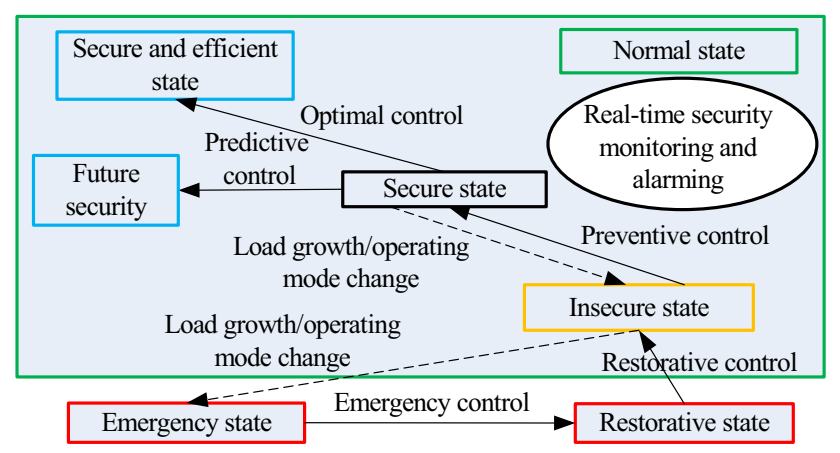

Fig. 1 Security states of distribution systems
5) Restorative state. The system situation is a transition to normal state after a series of power service restoration measures. Usually this is the aftermath of an emergency.

In Fig. 1, five security control approaches are also presented to describe the conversion of five states. The five control approaches are all proposed based on DSSR theory, and, the detailed function is discussed in section 4 .

\section{Framework with advanced security control}

The paper expands new functions for DMS in advanced security control, of which framework is shown in Fig. 2.

\subsection{Basic functions}

The basic functions of the management system consists of real-time data acquisition, basic data reduction, topology analysis, network connection modes identification, state estimation, power flow calculation, short circuit calculation, short-term load forecasting, load management, reliability analysis, $N-1$ simulation and other traditional basic function. Moreover, we strengthen the basic functions with some new security analysis functions such as real-time security distance calculation, security boundary visualization and calculation of output range of DGs. The new security analysis approaches are explained in detail as follows.

1) Real-time security distance calculation. The minimum distance from the operating point to all the security boundaries and overall weighted distance are calculated based on real-time load data [16]. DSSR boundary can be calculated in advance based on the topology and without considering load information. Once the boundary is determined, simple SD calculation without power flow will be enough for security assessment.

2) Security boundary visualization. Operators cannot directly observe the locations of operating points in DSSR, because the entire security boundary is a hyperplane, of which dimensions is commonly high. To visualize the location of the operating point, dimension-reduction security boundary figures will be drawn. We first find out components failing in $N-1$ test and its relative components as variables so that the dimension of DSSR can be reduced to 2 or 3 . With the dimension-reduction security boundary figures, operators can obtain security margins in each direction intuitively to perform security controls [17].

3) Security boundary of output range of DGs. The security distance of feeder loads can be used as 

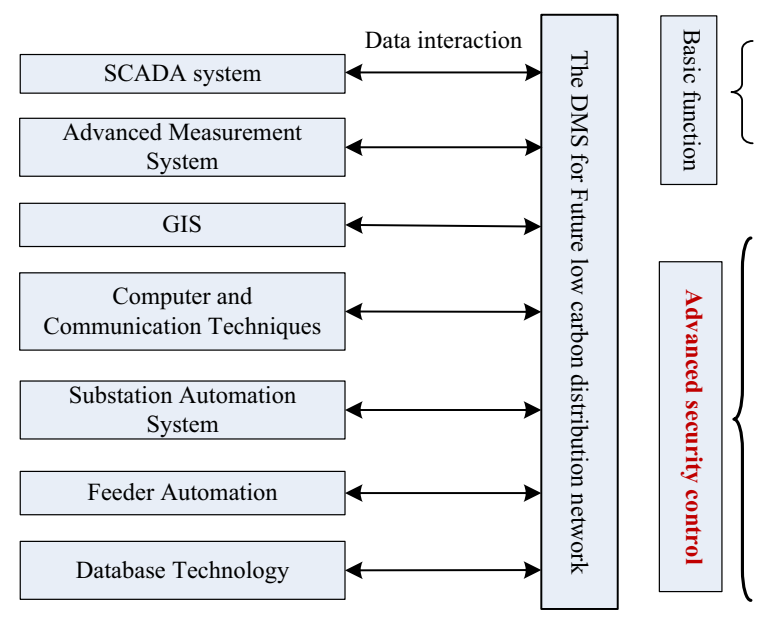

Traditional basic function

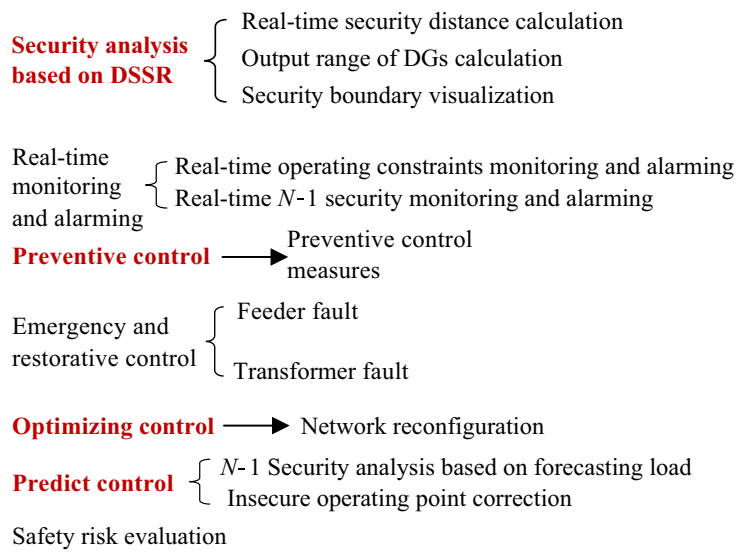

Fig. 2 The expansion of DMS framework with security functions

reference to determine the minimum output of DGs. Besides, the security region of DGs can be obtained, which is used to dispatch DGs' output limit.

New security analysis method based on DSSR is superior in calculation speed and it also can give operators more information about security operation, which is very suitable to be applied on line. Though the traditional $N-1$ simulation is time-consuming, it can discover the detailed data of $N-1$ contingency and form fault treatment measures. Meanwhile, the traditional $N-1$ simulation can verify the new security analysis approaches. These two methods are mutually complementary.

\subsection{Advanced security control}

1) Real-time monitoring and alarming. The problems and hidden threats would be monitored and alarmed based on real-time data in this function. Detailed procedures are conducted as follows.

a) Real-time operating constraints monitoring and alarming. If any operating parameter is out of limits, operators should receive warnings and take measures immediately. It should be pointed out that $N-1$ criteria are not considered in this procedure.

b) Real-time $N-1$ security monitoring and alarming. The previous procedure can't monitor hidden threats, which may lead to load shedding after a rational contingency. While this procedure can predetermine the DSSR boundary and calculate security distance online, by which operators can judge the system $N-1$ security. If the system is not secure, an alert message would be given. Meanwhile the $N-1$ simulation will be activated to find out the components not passing $N-1$ test and to obtain detailed fault data.

2) Preventive control. Once the system becomes insecure, the feeders or transformers, of which the security distance is negative, would be found out immediately through real-time monitoring and alarming. Then, $N-1$ simulation for these components will be activated. If necessary, dimension-reduction security boundary figures of these feeders or transformers will be drawn. Preventive controls will be taken according to the two results above. After the preventive control, the system becomes stronger when faced with any rational contingency.

3) Predictive control. Predictive control can help operators master the security development trend of the system and guide the system away from the dangerous operating region. First, the trajectory of operating points is tracked and forecasted in different time scales. Then, based on the load development trend and security distance calculation, security development trend is obtained. Finally, if the operating point is predicted to overflow the DSSR in the future, predict control will be implemented to correct its development trend. In other word, predict control ensures the system always operating inside the DSSR.

4) Optimizing control. Even if the operating point is secure, it could still be optimized further. When local network is heavy-load-carrying, hidden dangers for security operating such as little security margin, load disequilibrium and operating parameters that almost meet their limits would appear. Since these problems, optimizing control should be made, which resets open point of loop structure. By this way, load can be redistributed well while maintaining enough security margins. Different from traditional model to optimize 
nodal voltages and network losses mainly, the objective functions of the new optimizing control model also include security distance and load equilibrium.

5) Emergency and restorative control. When fault occurs at substation transformer, processing in the new management system would be different from traditional way. The traditional way is designed for current distribution automation. In tradition, auto-switch-on device would act immediately, so load of faulted transformer is firstly transferred to backup transformers in the same substation through bus connection switch. Here, overloading rate of the backup transformer less than 1.3 within limit time is permitted. And load should be transferred to other substations through linked feeders in 2 hours until the backup transformer is no longer overloaded. But if overloading rate is greater than 1.3, all load of faulted transformer would be cut off. Because overloading rate greater than 1.3 is usually considered too high and auto-switch-on device will lock itself automatically [18]. With large-scale distribution automation initiatives, transferring load among substations becomes much faster so that transformer overloading duration could decrease to minute level. Thus transformer can run with a higher loading level under the $N-1$ criteria. As for this management system, a maximum load capacity and security operation boundary considering the $N-1$ criteria can be calculated based on DSSR respectively, which can greatly improve system operation efficiency.

6) Safety risk evaluation. Probabilities of any single fault and security influences of faults are different. So precise safety risk evaluation takes fault probabilities, risk acceptances of users and operators into consideration. Based on the bearing degree of risks, the DSSR boundary would be expanded to exploit power supply potentiality of the distribution system. Moreover, the security level of the whole power network and key component can be evaluated and obtained by identifying different kinds of insecure factors and fault probabilities. Thus through selective equipment maintenance, fault probability and influence would be reduced. In other words, the risk would be managed. Meanwhile, safety risk evaluation also provides detailed information for reforming and programming the distribution network.

\section{Case study}

In this section, the proposed DSSR-based DMS is demonstrated on a real medium-voltage distribution system of one city in South China.
This system consists of 12 substations, 26 substation transformers and 114 feeders, as is shown in Fig. 3. Total capacity of substation transformers is 1094.5 MVA. All main feeders form single loop structure with a normally open switch; the whole medium-voltage network is completely upgraded with distribution automated (DA). The substation transformers and feeders are numbered in Fig. 3. $T_{i}$ represents substation transformer $i$ and $F_{i}$ represents feeder $i$.

\subsection{Calculation of DSSR boundary}

The dimension of the operating point and the number of sub-formulas of DSSR boundary are both 114, because the feeder scale of the test case is 114. The expression for DSSR boundary [17] is calculated and shown in the Appendix.

\subsection{Preventive control}

Take the operating point $W_{A}$ as an example. Security distances are calculated according to the method in literature [17], which is listed in Table B1 in the Appendix.

In the SD (security distance) calculation result, the number of negative SD is 4 , and its corresponding feeders are $F_{8}, F_{16}, F_{28}$ and $F_{40}$. Thus, $W_{A}$ is insecure. The 4 feeders above and corresponding substation transformers $\left(T_{2}, T_{4}, T_{6}, T_{8}\right)$ all fail to pass the $N-1$ test. Based on the topology, we obtain that the feeders with negative SD form link structure with the feeders of $T_{7}$ respectively, which means $T_{7}$ is the back-feed source for these feeders in the post-fault network. Therefore, overloading of $T_{7}$ could lead to the $N-1$ test fail. We visualize the 2D DSSR boundaries which describe the relationship of $F_{36}$ (from $T_{7}$ ) and relative feeders, including $F_{8}, F_{16}, F_{26}, F_{40}$ and $F_{56}$, as is shown in Fig. 4.

It is shown in Fig. 4 that $W_{A}$ is out of the security boundaries B1-B4. For this problem, it is found that $F_{8}$, $F_{16}, F_{28}$ and $F_{40}$ can go back into the security region after reducing the load of $F_{36}$, which will cause outage of some loads. Then, we can reduce the $F_{36}$ and increase $F_{56} . F_{36}$ and $F_{56}$ form a loop structure, among which load can be redistributed via various selection of open-loop point, as is shown in Fig. 5.

As is shown Fig. 5, the load of $F_{56}$ and $F_{36}$ can be relocated by disconnecting switch $\mathrm{K}_{6}$ and closing $\mathrm{K}_{5}$. Then, load of $F_{36}$ reduces to 5.500 MVA by 2.000 MVA, meanwhile, load of $F_{56}$ increases to 4.000 MVA by 2.000 MVA. After these preventive controls, the operating point $W_{A}$ outside the security boundary is adjusted to $W_{B}$, which is inside the security region. The data for $W_{B}$ is shown in Table B3 in the Appendix and the operating point $W_{B}$ passes the $N-1$ test. 


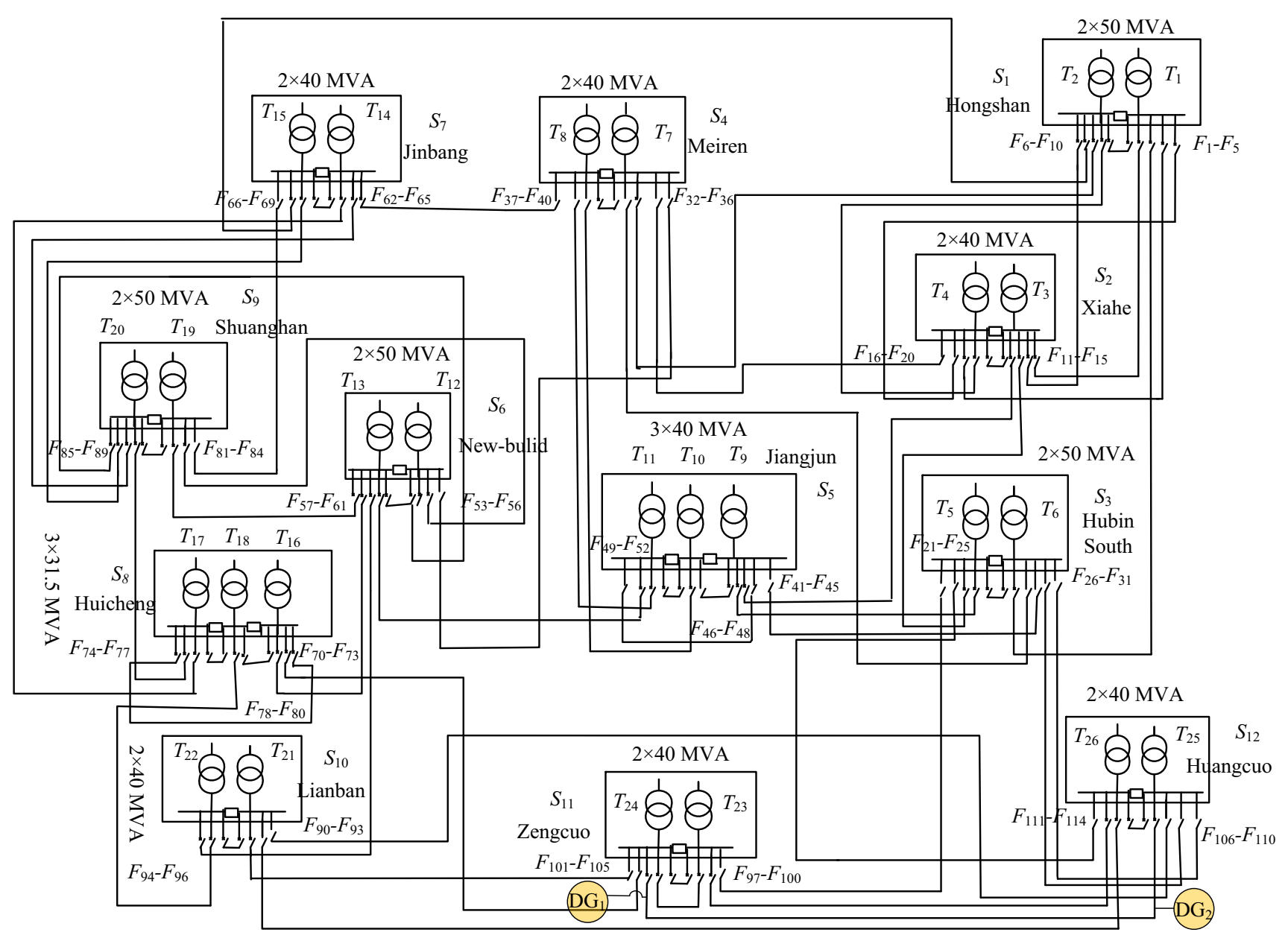

Fig. 3 Network configuration of a real system in a South China city

From Fig. 4 and Fig. 5, it can be seen that insecure operating point can be adjusted back into security region by re-distributing load in preventive control process.

\subsection{Predictive control}

Assume a practical event in summer workday peak load hours to illustrate the predictive control. $W_{C}$ is the operating point of test case at 8:00 a.m. $W_{D}$ is the operating point that $W_{C}$ will operate to in the peak load at 14:00 p.m. $W_{D}$ is 1.5 times of $W_{C}$ in the quantity of each feeder load. By security assessment, $W_{D}$ is an insecure point. To avoid the operating point outside the security region, dispatchers should adjust the $W_{C}$ to $W_{C}{ }^{\prime}$ at 8:00 a.m., by which the insecure $W_{D}$ will be adjusted to secure $W_{D}{ }^{\prime}$ at 14:00 p.m. The control above is based on such method: current operating point has effects on the future one, so measures for current operating point should be taken to induce the future one to the state with larger security margin.

A 3D DSSR section is shown in Fig. 6 to illustrate the control process above. Load profile, distance calculation
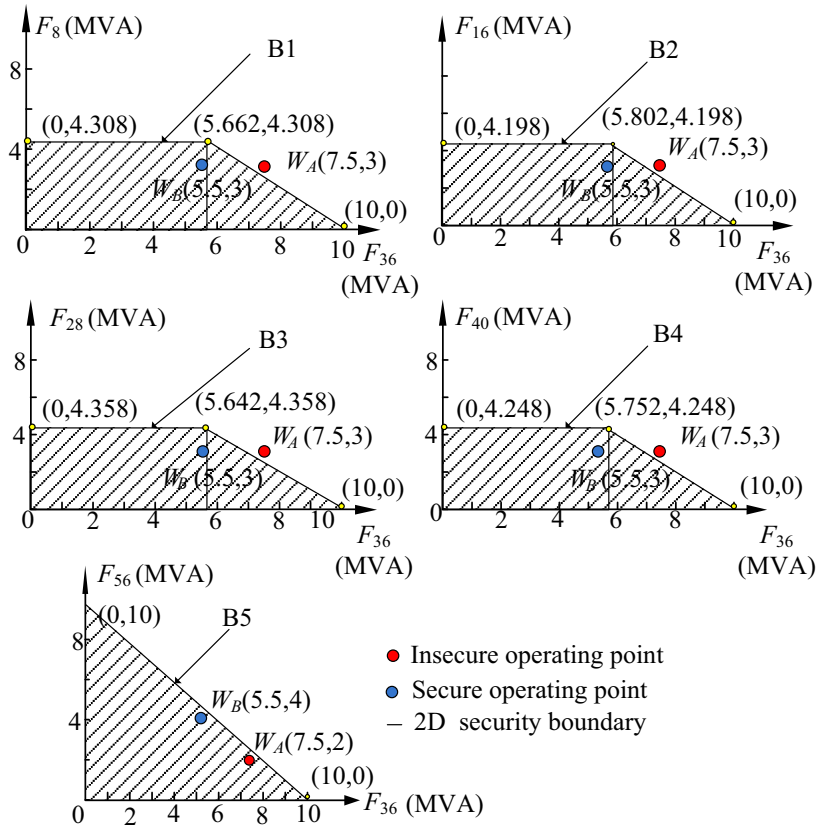

Fig. 4 The dimension reduction figure of security boundary 


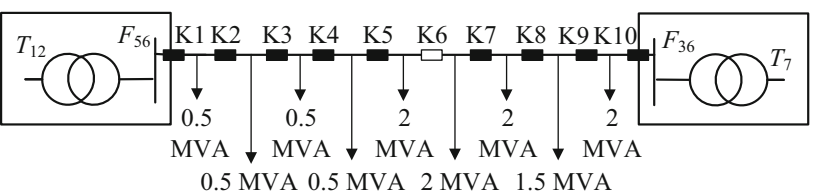

Fig. 5 Load re-distribution by network reconfiguration

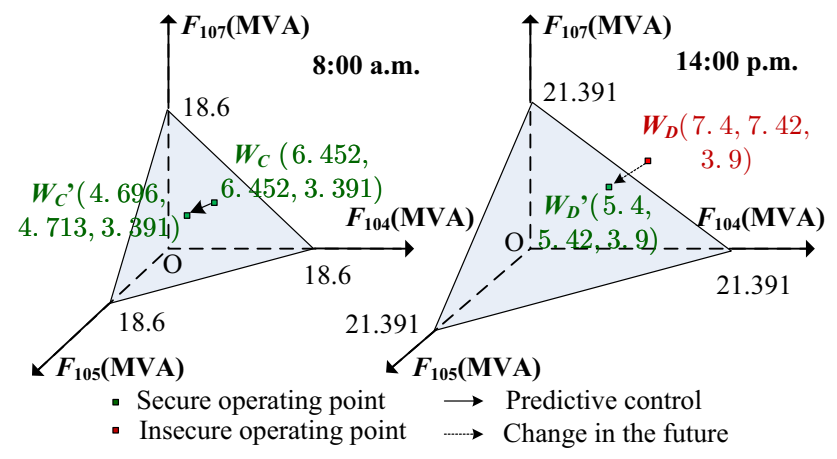

Fig. 6 Illustration of predictive control

and $N-1$ test result of operating points $W_{C}, W_{D}, W_{C}{ }^{\prime}, W_{D}{ }^{\prime}$ are shown in Table $\mathrm{C} 1$, Table $\mathrm{C} 2$ and Table $\mathrm{C} 3$ in the Appendix, the operating points $W_{C}, W_{C}{ }^{\prime}$ and $W_{D}{ }^{\prime}$ all pass the $N-1$ test, so $N-1$ security verification result tables are omitted.

\subsection{Optimal control}

In this section, we take the operating point $W_{E}$ as an example, of which load distribution is unbalanced. Loading rate of $T_{2}, T_{7}$ and $T_{24}$ is over-high and respectively reaches at $70 \%, 83.75 \%, 83.75 \%$. Additionally, the load profile and distance calculation of operating point $W_{E}$ are shown in Table D1 in the Appendix. Thus, some load should be transferred form high-ratio transformers to others. By this means, the load of system can fit with the network structure better, which optimizes the operation within security

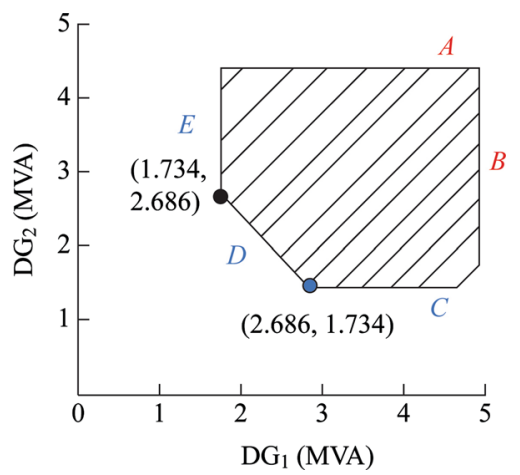

Fig. 7 Secure output range of $\mathrm{DG}_{1}$ and $\mathrm{DG}_{2}$

constrains. Here, $W_{E}$ is adjusted to $W_{F}$ by network reconfiguration. The complete load profile and distance calculation of $W_{F}$ are shown in Table D2 in the Appendix. Table 1 shows the comparison before and after optimal control.

From the Table 1, we can see that the minimum distance security is increased and the load equilibrium of substation transformers and feeders is also improved to maintain enough security margins by optimizing control.

\subsection{Secure output range of DGs}

Take operating point $W_{F}$ as an example, the complete load profile, SD calculation and $N-1$ test result of operating point $W_{F}$ are shown in Table E1 and Table E2 in the Appendix. The system is insecure without enough DGs output under current operating point, 4 feeders and 3 substation transformers fail in $N-1$ test. To ensure the system security, output of DGs should be kept in a range, as is shown in Fig. 7.

In Fig. 7, boundary $A$ and $B$ represent the upper limit of $\mathrm{DG}_{1}$ and $\mathrm{DG}_{2}$. Boundary $C, E$ represents the $N-1$ constrains. Boundary $D$ represents the power flow constrain between two DGs.

Table 1 Comparison before and after the optimal control

\begin{tabular}{|c|c|c|c|c|}
\hline $\begin{array}{l}\text { Operating } \\
\text { point }\end{array}$ & Equilibrium degree of feeder & Equilibrium degree of transformer & Sum of SD (MVA) & No. of negative SD \\
\hline$W_{E}$ & 14.314 & 31.486 & 124.906 & 0 \\
\hline$W_{F}$ & 9.724 & 17.475 & 126.708 & 0 \\
\hline $\begin{array}{l}\text { Operating } \\
\text { point }\end{array}$ & $\begin{array}{l}\text { Total load } \\
\text { (MVA) }\end{array}$ & $\begin{array}{l}\text { Minimum } \\
\text { value of } \\
\text { SD (MVA) }\end{array}$ & $\begin{array}{l}\text { Number of } \\
\text { failed } \\
\text { feeder } \\
N-1 \text { test }\end{array}$ & $\begin{array}{l}\text { No. of failed } \\
\text { transformer } \\
N-1 \text { test }\end{array}$ \\
\hline$W_{E}$ & 451.000 & 0.330 & 0 & 0 \\
\hline$W_{F}$ & 451.000 & 0.466 & 0 & 0 \\
\hline
\end{tabular}




\section{Features of new DMS based-on DSSR}

Compared with traditional distribution automation, the new DMS has following features.

1) Regular distribution automation is passive and focused on the post-fault state. When faced with peak load, load shedding and power consumption limitation may often occur In contrast, solution in this paper is active and capable to monitor the hidden threats in advance and then to take necessary preventive or predictive controls to guarantee that loads can be transferred without violating any constraints in case of any $N-1$ contingency. In other words, these controls ensure that operating points are always inside the security region. Thus, the system security and reliability can be improved significantly.

2) The operation mode based on distribution automation is focused on local feeder automation and do not make full use of the connection between substations. So the secure loading level for substation transformers is conservative. The new operation mode based on the proposed management system makes full use of connection between substations. The new idea is to combine the network transfer capability and substation supply capability, which results in a higher loading rate and better assets utilization within the $N-1$ criteria.

3) As for security assessment, the existing approaches are based on $N-1$ simulation. This case by case simulation approach is inferior in calculation speed to be applied on-line in large-scale grid. The new operation mode based on security region can precisely predetermine the security boundary and assess security much faster, which makes real-time security monitoring possible.

The proposed extended DMS framework is conceptually similar to Dy Liacco security framework for transmission system. However, they are different in control strategies and methods. Compared to transmission system, distribution system has lower security requirements and cares about only static security instead of transient security. Meanwhile, Distribution system is constructed in closed loop but operates radially, while the transmission network operates in closed loop. These contribute to more complex security region in transmission system. Also, [18] shows that distribution security boundary is approximately linear. Thus, it is easier to calculate the secure distance, which results in more convenient to realize security monitor and control in distribution systems.

\section{Conclusion}

This paper proposes a new DMS framework based on security region for future active distribution systems under low carbon background. This new approach aims to upgrade existing DMS, featuring with following characteristics:

1) Preventive and predictive security controls are easy to implement and provide active operational capability for distribution systems.

2) On-line security analysis is available based on $N-1$ security region.

3) The output range of DGs in the system security boundary can be calculated.

4) Good economic benefit is gained through higher loading rate within same security standard.

A practical case is presented to illustrate the proposed DSSR-based DMS. The security region of both networks and DGs are calculated and the real-time security controls are demonstrated. It is shown that the new DMS framework can improve the efficiency and ensure the security of distribution systems.

This paper shows good prospects for the application of DSSR theory in future DMS. However, there are many works need to do, such as upgraded models and methods considering demand response and electric vehicles.

Acknowledgement This work was supported by the National Natural Science Foundation of China (51477112) and National Natural Science Foundation of China (51277129).

Open Access This article is distributed under the terms of the Creative Commons Attribution 4.0 International License (http:// creativecommons.org/licenses/by/4.0/), which permits unrestricted use, distribution, and reproduction in any medium, provided you give appropriate credit to the original author(s) and the source, provide a link to the Creative Commons license, and indicate if changes were made.

\section{Appendix}

Please note that only the important data are listed because of the limited space. If you need more data, please contact with the authors. 
Partial expression of security region boundary

$$
\begin{aligned}
& \left\{\begin{aligned}
L_{F_{1}} \leq & \min \left[11.778-L_{F_{10}}, 50-\right. \\
& \left.\left(L_{F_{6}}+L_{F_{7}}+L_{F_{8}}+L_{F_{9}}+L_{F_{10}}\right)\right]
\end{aligned}\right. \\
& L_{F_{2}} \leq \min \left[11.778-L_{F_{15}}, 40-\right. \\
& \left.\left(L_{F_{11}}+L_{F_{12}}+L_{F_{13}}+L_{F_{14}}+L_{F_{15}}\right)\right] \\
& L_{F_{3}} \leq \min \left[11.778-L_{F_{27}}, 50-\right. \\
& \left.\left(L_{F_{26}}+L_{F_{27}}+L_{F_{28}}+L_{F_{29}}+L_{F_{30}}+L_{F_{31}}\right)\right] \\
& L_{F_{4}} \leq \min \left[11.778-L_{F_{18}}, 40-\right. \\
& \left.\left(L_{F_{16}}+L_{F_{17}}+L_{F_{18}}+L_{F_{19}}+L_{F_{20}}\right)-L_{F_{5}}\right] \\
& L_{F_{5}} \leq \min \left[11.778-L_{F_{17}}, 40-\right. \\
& \left.\left(L_{F_{16}}+L_{F_{17}}+L_{F_{18}}+L_{F_{19}}+L_{F_{20}}\right)-L_{F_{4}}\right] \\
& L_{F_{6}} \leq \min \left[11.778-L_{F_{14}}, 40-\right. \\
& \left.\left(L_{F_{11}}+L_{F_{12}}+L_{F_{13}}+L_{F_{14}}+L_{F_{15}}\right)\right] \\
& L_{F_{7}} \leq \min \left[11.778-L_{F_{67}}, 40-\right. \\
& \left.\left(L_{F_{66}}+L_{F_{67}}+L_{F_{68}}+L_{F_{69}}\right)\right] \\
& B_{D S S R}= \\
& L_{F_{108}} \leq \min \left[10.566-L_{F_{93}}, 40-\right. \\
& \left.\left(L_{F_{90}}+L_{F_{91}}+L_{F_{92}}+L_{F_{93}}\right)\right] \\
& L_{F_{109}} \leq \min \left[11.778-L_{F_{30}}, 50-\right. \\
& \left.\left(L_{F_{26}}+L_{F_{27}}+L_{F_{28}}+L_{F_{29}}+L_{F_{30}}+L_{F_{31}}\right)-L_{F_{110}}\right] \\
& L_{F_{110}} \leq \min \left[11.778-L_{F_{31}}, 50-\right. \\
& \left.\left(L_{F_{26}}+L_{F_{27}}+L_{F_{28}}+L_{F_{29}}+L_{F_{30}}+L_{F_{31}}\right)-L_{F_{109}}\right] \\
& L_{F_{111}} \leq \min \left[11.778-L_{F_{22}}, 50-\right. \\
& \left.\left(L_{F_{21}}+L_{F_{22}}+L_{F_{23}}+L_{F_{24}}+L_{F_{25}}\right)\right] \\
& L_{F_{112}} \leq \min \left[10.566-L_{F_{99}}, 40-\right. \\
& \left.\left(L_{F_{97}}+L_{F_{98}}+L_{F_{99}}+L_{F_{100}}\right)\right] \\
& L_{F_{113}} \leq \min \left[10.566-L_{F_{92}}, 40-\right. \\
& \left.\left(L_{F_{90}}+L_{F_{91}}+L_{F_{92}}+L_{F_{93}}\right)\right] \\
& L_{F_{114}} \leq \min \left[10.566-L_{F_{106}}, 40-\right. \\
& \left.\left(L_{F_{106}}+L_{F_{107}}+L_{F_{108}}+L_{F_{109}}+L_{F_{110}}\right)\right]
\end{aligned}
$$

\begin{tabular}{|c|c|c|}
\hline $\begin{array}{l}\text { Number of connected } \\
\text { transformers }\end{array}$ & $\begin{array}{l}\text { Number of feeder } \\
\text { connections }\end{array}$ & $\begin{array}{l}\text { Feeder capacity } \\
\text { (MVA) }\end{array}$ \\
\hline $5-6$ & 1 & 11.778 \\
\hline $5-9$ & 1 & 11.778 \\
\hline $5-23$ & 1 & 11.778 \\
\hline $5-26$ & 1 & 11.778 \\
\hline $6-7$ & 1 & 11.778 \\
\hline $6-9$ & 1 & 11.778 \\
\hline $6-25$ & 2 & 11.778 \\
\hline $7-8$ & 1 & 11.778 \\
\hline $7-12$ & 1 & 10.566 \\
\hline $8-10$ & 1 & 10.566 \\
\hline $8-11$ & 1 & 10.566 \\
\hline $8-14$ & 1 & 10.566 \\
\hline $9-10$ & 1 & 10.566 \\
\hline 9-11 & 1 & 10.566 \\
\hline $10-11$ & 1 & 10.566 \\
\hline $11-13$ & 1 & 10.566 \\
\hline $12-13$ & 1 & 10.566 \\
\hline $12-19$ & 1 & 10.566 \\
\hline $12-20$ & 1 & 10.566 \\
\hline $13-16$ & 1 & 10.566 \\
\hline 13-19 & 1 & 10.566 \\
\hline $13-22$ & 1 & 10.566 \\
\hline $14-15$ & 1 & 10.566 \\
\hline $14-17$ & 1 & 10.566 \\
\hline $14-20$ & 1 & 10.566 \\
\hline $15-19$ & 1 & 10.566 \\
\hline $15-20$ & 1 & 10.566 \\
\hline $16-17$ & 1 & 10.566 \\
\hline $16-18$ & 1 & 10.566 \\
\hline $16-24$ & 1 & 10.566 \\
\hline $17-18$ & 1 & 10.566 \\
\hline $17-20$ & 1 & 10.566 \\
\hline $18-22$ & 1 & 10.566 \\
\hline 19-20 & 1 & 10.566 \\
\hline $21-22$ & 1 & 10.566 \\
\hline $21-24$ & 1 & 10.566 \\
\hline $21-25$ & 1 & 10.566 \\
\hline $21-26$ & 1 & 10.566 \\
\hline $23-24$ & 2 & 10.566 \\
\hline $23-26$ & 1 & 10.566 \\
\hline $24-25$ & 1 & 10.566 \\
\hline $25-26$ & 1 & 10.566 \\
\hline
\end{tabular}

Table A1 Capacity of transformer links

\begin{tabular}{lll}
\hline $\begin{array}{l}\text { Number of connected } \\
\text { transformers }\end{array}$ & $\begin{array}{l}\text { Number of feeder } \\
\text { connections }\end{array}$ & $\begin{array}{l}\text { Feeder capacity } \\
\text { (MVA) }\end{array}$ \\
\hline $1-2$ & 1 & 11.778 \\
$1-3$ & 1 & 11.778 \\
$1-4$ & 2 & 11.778 \\
$1-6$ & 1 & 11.778 \\
$2-3$ & 1 & 11.778 \\
$2-4$ & 1 & 11.778 \\
$2-7$ & 1 & 11.778 \\
$2-15$ & 1 & 11.778 \\
$3-4$ & 1 & 11.778 \\
$3-5$ & 1 & 11.778 \\
$3-9$ & 1 & 11.778 \\
$4-7$ & 1 & 11.778 \\
\hline
\end{tabular}

Table A1 continued 
Table B1 Load and security distance of feeders before the prevent control

\begin{tabular}{lll}
\hline Number & Load (MVA) & SD (MVA) \\
\hline F8 & 3.33 & -0.830 \\
F16 & 3.62 & -1.120 \\
F28 & 3.23 & -0.730 \\
F32 & 7.53 & 0.448 \\
F33 & 7.42 & 1.128 \\
F34 & 7.47 & 0.978 \\
F35 & 7.58 & 0.578 \\
F36 & 7.50 & 1.066 \\
F40 & 3.80 & -1.300 \\
F56 & 2.00 & 0.500 \\
$\cdots$ & $\ldots$ & $\cdots$ \\
\hline
\end{tabular}

Table B2 $N-1$ security verification of feeders and transformers before the prevent control

\begin{tabular}{llll}
\hline Number & $N-1$ result & Number & $N-1$ result \\
\hline F8 & 0 & F28 & 0 \\
T2 & 0 & T6 & 0 \\
F16 & 0 & F40 & 0 \\
T5 & 0 & T8 & 0
\end{tabular}

Note: Failed $N-1$ test of feeders and transformers are listed, but successful $N-1$ test for feeders and transformers are omitted

Table B3 Load and safety distance of feeders after the prevent control

\begin{tabular}{lll}
\hline Number & Load (MVA) & SD (MVA) \\
\hline F8 & 3.33 & 0.978 \\
F16 & 3.62 & 0.588 \\
F28 & 3.23 & 1.128 \\
F32 & 7.53 & 0.448 \\
F33 & 7.42 & 1.128 \\
F34 & 7.47 & 0.978 \\
F35 & 7.58 & 0.578 \\
F36 & 5.50 & 1.066 \\
F40 & 3.80 & 0.448 \\
F56 & 4.00 & 0.500 \\
$\ldots$ & $\ldots$ & $\cdots$ \\
\hline
\end{tabular}

Table C1 Load and safety distance of feeders of working point $W_{C}$ and $W_{D}$

\begin{tabular}{llll}
\hline Number & $\begin{array}{l}\text { Load of } \\
W_{C}(\text { MVA) }\end{array}$ & $\begin{array}{l}\text { SD of } W_{C} \\
\text { (MVA) }\end{array}$ & $\begin{array}{l}\text { SD of } W_{D} \\
\text { (MVA) }\end{array}$ \\
\hline F101 & 6.435 & 2.288 & 1.046 \\
F102 & 6.435 & 1.957 & 0.666 \\
F105 & 6.435 & 2.653 & 1.466 \\
\hline
\end{tabular}

Table $\mathbf{C 1}$ continued

\begin{tabular}{llll}
\hline Number & $\begin{array}{l}\text { Load of } \\
W_{C} \text { (MVA) }\end{array}$ & $\begin{array}{l}\text { SD of } W_{C} \\
\text { (MVA) }\end{array}$ & $\begin{array}{l}\text { SD of } W_{D} \\
\text { (MVA) }\end{array}$ \\
\hline F103 & 5.739 & 1.436 & 0.066 \\
F104 & 6.452 & 2.723 & 1.546 \\
F107 & 3.391 & 1.436 & -0.120 \\
\hline
\end{tabular}

Note: The load capacity of operating point $W_{D}$ is 1.15 times of that of operating point $W_{C}$. Detailed load information of $W_{D}$ is omitted

Table C2 $N-1$ security verification of feeders and transformers of operating point $W_{D}$

\begin{tabular}{ll}
\hline Number & $N-1$ result \\
\hline F107 & 0 \\
- & - \\
\hline
\end{tabular}

Table C3 Load and safety distance of feeders of operating point $W_{C}{ }^{\prime}$ and $W_{D}{ }^{\prime}$

\begin{tabular}{llll}
\hline Number & $\begin{array}{l}\text { Load of } W_{C}{ }^{\prime} \\
(\mathrm{MVA})\end{array}$ & $\begin{array}{l}\text { SD of } W_{C}{ }^{\prime} \\
(\mathrm{MVA})\end{array}$ & $\begin{array}{l}\text { SD of } W_{D}{ }^{\prime} \\
(\mathrm{MVA})\end{array}$ \\
\hline F101 & 6.435 & 2.288 & 1.046 \\
F102 & 6.435 & 1.957 & 0.666 \\
F105 & 4.696 & 2.653 & 1.466 \\
F103 & 5.739 & 1.436 & 0.066 \\
F104 & 4.713 & 2.723 & 1.546 \\
F107 & 3.391 & 1.436 & 0.066
\end{tabular}

Note: The load capacity of operating point $W_{D}{ }^{\prime}$ is 1.15 times of that of operating point $W_{C}{ }^{\prime}$. Detailed load information of $W_{D}{ }^{\prime}$ is omitted

Table D1 Load and safety distance of feeders before the optimal control

\begin{tabular}{lll}
\hline Number & Load (MVA) & SD (MVA) \\
\hline F1 & 3.12 & 0.658 \\
F6 & 8.00 & 0.888 \\
F7 & 8.03 & 0.628 \\
F9 & 7.84 & 1.048 \\
F10 & 8.00 & 0.658 \\
F14 & 2.89 & 0.888 \\
F16 & 3.12 & 1.368 \\
F19 & 2.89 & 1.048 \\
F28 & 3.03 & 1.748 \\
F32 & 7.40 & 0.578 \\
F33 & 7.00 & 1.748 \\
\hline
\end{tabular}


Table D1 continued

\begin{tabular}{lll}
\hline Number & Load (MVA) & SD (MVA) \\
\hline F35 & 7.30 & 1.358 \\
F36 & 6.80 & 0.766 \\
F40 & 3.80 & 0.578 \\
F46 & 4.80 & 1.936 \\
F47 & 5.20 & 1.566 \\
F48 & 5.00 & 2.446 \\
F56 & 3.00 & 0.766 \\
F67 & 3.12 & 0.628 \\
F72 & 3.00 & 0.566 \\
F91 & 3.12 & 0.446 \\
F97 & 3.04 & 0.330 \\
F98 & 3.13 & 0.330 \\
F101 & 7.00 & 0.446 \\
F102 & 7.00 & 0.566 \\
F103 & 7.00 & 0.536 \\
F104 & 6.50 & 0.936 \\
F105 & 6.00 & 1.526 \\
F107 & 3.03 & 0.536 \\
$\ldots$ & $\ldots$ & $\ldots$ \\
\hline
\end{tabular}

Table D2 Load and safety distance of feeders after the optimal control

\begin{tabular}{|c|c|c|}
\hline Number & Load (MVA) & SD (MVA) \\
\hline F1 & 5.12 & 0.658 \\
\hline F6 & 6.00 & 0.888 \\
\hline F7 & 6.03 & 0.628 \\
\hline F9 & 5.84 & 1.048 \\
\hline F10 & 6.00 & 0.658 \\
\hline F14 & 4.89 & 0.888 \\
\hline F16 & 5.12 & 1.368 \\
\hline F19 & 4.89 & 1.048 \\
\hline F28 & 5.03 & 1.748 \\
\hline F32 & 5.40 & 0.578 \\
\hline F33 & 5.00 & 1.748 \\
\hline F35 & 5.30 & 1.358 \\
\hline F36 & 4.80 & 0.766 \\
\hline F40 & 5.80 & 0.578 \\
\hline F46 & 5.00 & 1.736 \\
\hline F47 & 4.80 & 1.966 \\
\hline $\mathrm{F} 48$ & 5.20 & 2.246 \\
\hline F56 & 5.00 & 0.766 \\
\hline F67 & 5.12 & 0.628 \\
\hline F72 & 5.00 & 0.566 \\
\hline F91 & 5.12 & 0.446 \\
\hline F97 & 5.04 & 1.526 \\
\hline F98 & 5.13 & 0.936 \\
\hline
\end{tabular}

Table D2 continued

\begin{tabular}{lll}
\hline Number & Load (MVA) & SD (MVA) \\
\hline F101 & 5.00 & 0.446 \\
F102 & 5.00 & 0.566 \\
F103 & 5.00 & 0.536 \\
F104 & 4.50 & 0.936 \\
F105 & 4.00 & 1.526 \\
F107 & 5.03 & 0.536 \\
$\ldots$ & $\ldots$ & $\ldots$ \\
\hline
\end{tabular}

Table E1 Load and safety distance of feeders of $W_{F}$

\begin{tabular}{lll}
\hline Number & Load (MVA) & SD (MVA) \\
\hline F97 & 2.05 & -1.730 \\
F98 & 1.76 & -1.730 \\
F99 & 3.89 & 2.476 \\
F100 & 3.94 & 4.838 \\
F101 & 7.34 & 2.206 \\
F102 & 6.90 & 1.776 \\
F103 & 7.56 & -1.734 \\
F104 & 8.8 & 0.006 \\
F105 & 7.32 & 1.196 \\
F106 & 3.12 & 4.516 \\
F107 & 4.74 & -2.66 \\
F108 & 2.96 & 3.776 \\
F109 & 2.29 & 6.638 \\
F110 & 3 & 5.738 \\
$\ldots$ & $\ldots$ & $\ldots$ \\
\hline
\end{tabular}

Table E2 $N-1$ security verification of feeders and transformers of $W_{F}$

\begin{tabular}{ll}
\hline Number & $N-1$ result \\
\hline F97 & 0 \\
T23 & 0 \\
F98 & 0 \\
T24 & 0 \\
F103 & 0 \\
T25 & 0 \\
F107 & 0 \\
\hline
\end{tabular}

\section{References}

[1] Grubb M, Jamasb T, Pollitt MG (2008) Delivering a low-carbon electricity system: technologies, economics and policy. Cambridge University Press, London

[2] Gelmini A, Benini M, Borgarello M (2008) Assessment of the actions to reduce $\mathrm{CO}_{2}$ emissions in the Italian power system. In: Proceedings of the 5th international conference on European 
electricity market (EEM'08), Lisbon, Portugal, 28-30 May 2008, 5 pp

[3] Grubb M, Butler L, Twomey P (2006) Diversity and security in UK electricity generation: The influence of low-carbon objectives. Energy Policy 34(18):4050-4062

[4] Smallwood C, Wennermark J (2010) Benefit of distribution automation. IEEE Trans Ind Appl Mag 16(1):65-73

[5] Mamo X, Mallet S, Coste T et al (2009) Distribution automation: the cornerstone for smart grid development strategy. In: Proceedings of the 2009 IEEE power and energy society general meeting, Calgary, Canada, 26-30 Jul 2009, 6 pp

[6] Tan ZF, Ngan HW, Wu Y et al (2013) Potential and policy issues for sustainable development of wind power in China. J Mod Power Syst Clean Energy 1(3):204-215. doi:10.1007/ s40565-013-0037-8

[7] Ipakchi A, Albuyeh F (2009) Grid of the future. IEEE Power Energy Mag 7(2):52-62

[8] Jayaweera D, Islam S (2014) Steady-state security in distribution networks with large wind farms. J Mod Power Syst Clean Energy 2(2):134-142. doi:10.1007/s40565-014-0052-4

[9] Nick M, Cherkaoui R, Paolone M (2014) Optimal allocation of dispersed energy storage systems in active distribution networks for energy balance and grid support. IEEE Trans Power Syst 29(5):2300-2310

[10] Esau Z, Jayaweera D (2014) Reliability assessment in active distribution networks with detailed effects of PV systems. J Mod Power Syst Clean Energy 2(1):59-68. doi:10.1007/s40565-0140046-2

[11] Romero-Ramos E, Gómez-Expósito A, Marano-Marcolini A et al (2011) Assessing the loadability of active distribution networks in the presence of DC controllable links. IET Gener Transm Distrib 5(11):1105-1113

[12] Singh N, Kliokys E, Feldmann H et al (1998) Power system modelling and analysis in a mixed energy management and distribution management system. IEEE Trans Power Syst 13(3): 1143-1149

[13] Song I, Yun SY, Kwon S et al (2013) Design of smart distribution management system for obtaining real-time security analysis and predictive operation in Korea. IEEE Trans Smart Grid 4(1):375-382
[14] Meliopoulos APS, Polymeneas E, Tan ZY et al (2013) Advanced distribution management system. IEEE Trans Smart Grid 4(4):2109-2117

[15] Bucher MA, Vrakopoulou M, Andersson G (2013) Probabilistic $N-1$ security assessment incorporating dynamic line ratings. In: Proceedings of the 2013 IEEE power and energy society general meeting, Vancouver Canada, 21-25 Jul 2013, 5 pp

[16] Xiao J, Gu W, Wang C et al (2012) Distribution system security region: Definition, model and security assessment. IET Gener Transm Distrib 6(10):1029-1035

[17] Xiao J, Zu GQ, Gong XX et al (2014) Model and topological characteristics of power distribution system security region. J Appl Math 327078/1-13

[18] Xiao J, Gong XX, He QB et al (2014) Topological characteristics and algorithm of $N-1$ security boundary for smart distribution network. P CSEE 34(4):545-554 (in Chinese)

[19] Wu F, Kumagai S (1982) Steady-state security regions of power systems. IEEE Trans Circuits Syst 29(11):703-711

[20] (2006) Guidelines of urban power network planning. State Grid Corporation of China, Beijing, China (in Chinese)

Jun XIAO received his B.S., M.S and Ph.D degrees in Tianjin University, Tianjin, China, in 1994, 1997, 2003, respectively. Presently, he is a professor in School of Electrical Engineering and Automation at Tianjin University. His research interests include distribution systems planning, assessment and micro-grid planning.

Qibo HE received his B.S. degree in electrical engineering from Tianjin University, Tianjin, China in 2013. She is now working toward the M.S. degree in Tianjin University. Her research interests are distribution systems planning and assessment.

Guoqiang $\mathbf{Z U}$ received his B.S. degree in electrical engineering from Tianjin University, Tianjin, China in 2012. He is now working toward the Ph.D degree in Tianjin University. His research interests are distribution systems planning and assessment. 